Proceedings of the Institution of
Civil Engineers
Transport I58
February 2005 Issue TRI
Pages 45-5I
Paper 13502
Received $17 / 09 / 2003$
Accepted 31/08/2004
Keywords:
bitumen \& tar/roads \& highways/
strength \& testing of materials
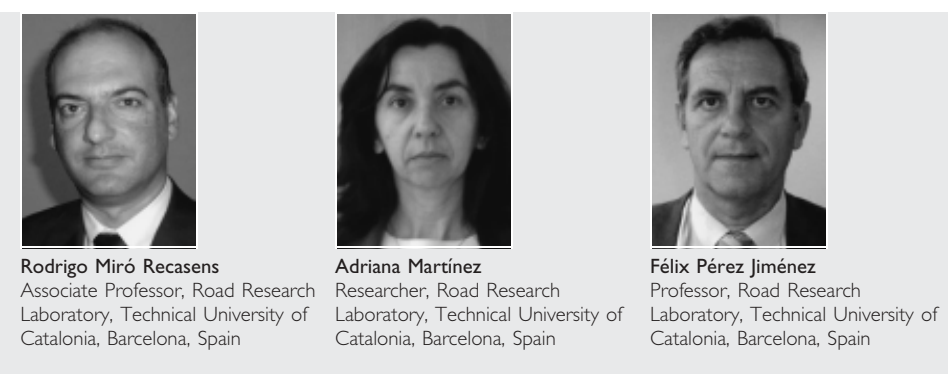

\title{
Assessing heat-adhesive emulsions for tack coats
}

R. Miró Recasens PhD, CEng, A. Martínez PhD, CEng and F. Pérez Jiménez PhD, CEng

The use of conventional emulsions for tack coats can cause problems as they frequently stick to the tyres of construction vehicles. Consequently, the bond between the asphalt layers is inadequate. Recently, new types of emulsions have been developed from very lowpenetration bitumen that contain no flux. They are known as 'heat-adhesive' emulsions and they are resistant to construction vehicles. However, the adhesive ability of these new emulsions has not previously been studied closely, particularly at low temperatures. The purpose of this study is to analyse the effect of different heat-adhesive emulsions and to verify their performance in service in comparison with the response of a conventional emulsion. For this reason a new shear test-the LCB test-has been developed. This test is carried out at different temperatures, both on laboratory specimens and on cores extracted from recently constructed pavements, where the same emulsions and dosages have been used. It is the modified heat-adhesive emulsion that performs best over the selected range of temperatures. The conventional heatadhesive emulsion, while performing well at medium temperatures, does not achieve the same resistance at the other temperatures as it is more temperature susceptible.

\section{INTRODUCTION}

In a pavement project, it is assumed that all the asphalt layers are going to work together; that is to say, that there is a good bond between the different layers. In order to obtain such a bond tack coats are applied during construction. However, experience has shown that the required bond is not always obtained, causing deterioration and early failure in the pavement.

The reason for this is that the final bond achieved between the layers depends on many factors: the type of emulsion used, the quantity of emulsion applied, the method of application, the adherence of the coat to the layer below, the possible effect of sticking to tyres of construction vehicles, how the new layer is constructed, the compaction level of the lower part of this layer, the mastic quality of the overlying layer, and so on.

The importance of tack coats in the performance of the pavement means that bituminous emulsions are constantly being improved. Recently, new types of emulsions have been developed from very low-penetration bitumen that contain no flux. They are known as heat-adhesive emulsions and are resistant to construction vehicles. Nevertheless, at present the adhesive ability of these new emulsions has not been studied closely, particularly at low temperatures.

The test procedures which have previously been used to assess the degree of adhesion between two asphalt layers using a tack coat can be divided into two main types: tensile and shear.

In direct tensile tests, clamps have to be tightened or attached directly onto the material that makes up each of the layers. This may be difficult to do correctly without causing eccentric loading, which would distort the test, and special clamps have to be made precisely for this purpose. These problems could be avoided if indirect rather than direct tensile tests were used. The wedge splitting test, developed by Tschegg et al., ${ }^{1,2}$ can be used to obtain a load-displacement curve. This is achieved by applying a force to a dual-layer specimen, by pushing a wedge into the interface between the two layers until they separate as a result of the horizontal component of the force.

The second group of tests used to evaluate the bond between layers are direct shear tests. Uzan et al., ${ }^{3}$ of Technion I.I.T. in Haifa, Israel, undertook a practical study of the importance of tack coats. They used a type of direct shear test on a dual-layer system of asphalt mixes, varying the temperature, the quantity of tack coat and the vertical force that was applied to the specimen during the test.

Caltabiano and Brunton ${ }^{4}$ of Nottingham University used, among other tests, the shear box test developed by Hughes, ${ }^{5}$ in a study on the reflection or propagation of cracks in the asphalt layers of pavements. In a similar way, Molenaar ${ }^{6}$ used the direct shear test of Delft University of Technology to assess the shear force and the shear stiffness index of an anticracking membrane between two layers of a mix made following the Marshall procedure (101.4 mm diameter specimens) and applying a load at $50 \cdot 8 \mathrm{~mm} / \mathrm{min}$. The same test procedure is found in the draft copy of the German specification 'Testing of layered composite according to Leutner, ${ }^{7}$ applied to $150 \mathrm{~mm}$ diameter cores.

More recently, Abdo et al. ${ }^{8}$ have adapted a direct shear test (US/Belge) on cylindrical specimens, with the specimen dimensions normally used in France (100 mm diameter). In this 
test the two layers that make up the specimen are held by cylindrical clamps and subjected to a tensile force, at a constant load speed of $25 \mathrm{kN} / \mathrm{min}$. The specimens are taken from blocks compacted in the Laboratoire des Ponts et Chausées (LPC) compactor using moulds that can be dismantled. The test, currently available for Laboratoire Régional de l'Ouest Parisien (LROP), was applied in the study of the bond of a bituminous mixture for fine layers (BBTM, in French terminology) on a layer of concrete, which had been made a few days earlier.

Another new type of test has been developed to determine the bond strength between a thin surfacing system and its substrate. At the required temperature a shearing torque is applied to a metal plate bonded to the surface of the top layer. This procedure, called the torque bond test, has been issued as a draft for development by the British Board of Agrément ${ }^{9}$ (according to Khweir and Fordyce ${ }^{10}$ ).

One of the first conclusions to be drawn from a review of the procedures and tests currently used to assess the adhesion between layers is that there is no one clear and simple procedure that is used by researchers.

For this reason the Road Research Laboratory of the Department of Transportation of the Technical University of Catalonia has developed a new shear test: the LCB (Laboratorio de Caminos de Barcelona) shear test ${ }^{11,12}$ which, unlike existing tests, is better suited to the equipment and the testing procedures used in Spain. This test is very simple to perform and capable of measuring resistance to tangential stresses caused by a shear force applied to the bond between the two layers.

This test allows both the assessment of the effect of several types of tack coats by testing specimens made in the laboratory, and also the quality control in works by testing the cores. The purpose of this work is to analyse the mechanical response of tack coats while using heat-adhesive emulsions and to verify the behaviour in service, comparing it with the response of a conventional emulsion.

To this end, three types of emulsion were chosen: conventional heat-adhesive emulsion, modified heat-adhesive emulsion and conventional emulsion. The LCB shear test was applied at different temperatures, both dry and after water immersion, and both on laboratory specimens and on cores extracted from the recently constructed pavements where the same emulsions and dosages were used.

\section{LCB SHEAR TEST}

The new LCB shear test that has been developed is based on the experiences mentioned above and aims to simplify the measurement procedure. It is intended to measure the resistance to tangential stresses, caused by the application of shear force, which is produced in the bond between the two asphalt layers whether or not a tack coat has been used. The deformation or the displacement of one layer with respect to the other is also measured.

In the test, shear stress is generated in the bond of the asphalt layers to be tested, until it separates them. The specimen is then used as a beam located over two supports, with the test section very close to one of the supports so that the bending moment is almost zero and the specimen is effectively only subjected to shear stress (Fig. 1).

\section{I. Testing of specimens: design of tack coats}

The specimens manufactured in the laboratory consist of two asphalt layers between which the tack coat to be studied is applied. This dual layer system is prepared in a cylindrical mould $177.8 \mathrm{~mm}$ high and with an interior diameter of $101.6 \mathrm{~mm} .^{13}$ The asphalt layers are compacted with a Marshall Compactor by applying 100 compaction blows to the upper surface. Once the first layer, of about 10 or $11 \mathrm{~cm}$ in height, is compacted, this should be left to cool.

Before applying the tack coat, the upper part of the specimen is sawn off so that the tack coat is applied to a surface without asphalt mortar, which can improve the bond between layers. Placing fresh material on a recently made layer is not the same as placing it on a worn layer, which has no bitumen on its surface. Therefore the specimen is cut by positioning it in the mould so that it is partly protruding. The upper part is sawn off and then the specimen is repositioned so that the tack coat can be applied and the next layer can be compacted. Before adding the second layer, the required quantity of emulsion to be assessed is applied with a brush and then left until the emulsion breaks.

A brush is used so that the application of the emulsion on the surface of the specimen is uniform. The quantity of emulsion used is determined from the difference between the weight of the specimen before and after the application.

Next, the hot bituminous mixture used for the top layer is poured on and compacted. Finally, the specimen is partially

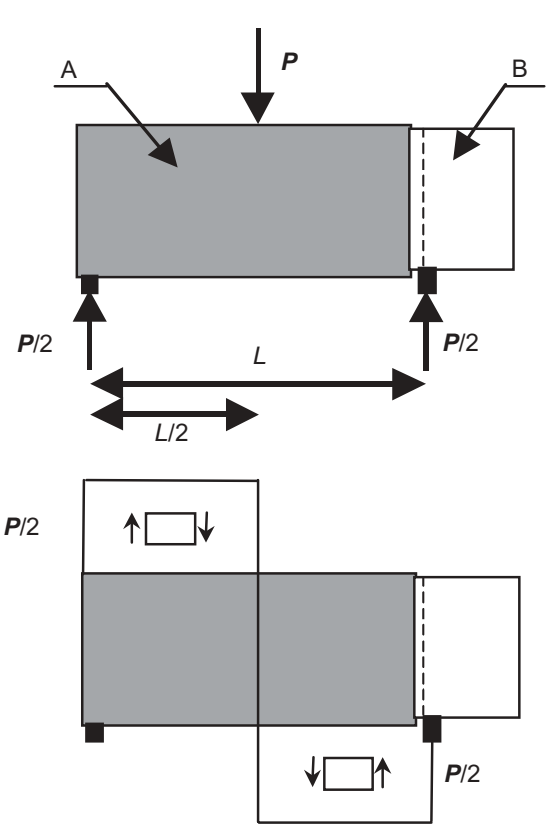

Fig. I. Diagram of forces and stresses acting on the specimen in the LCB shear test 
extruded from the mould so that the joint between the layers is $5 \mathrm{~mm}$ beyond the top of the mould.

The test is performed by placing the mould with the specimen inside horizontally over a base prepared with two supports $20 \mathrm{~cm}$ apart. The mould rests on one support and the upper layer of the specimen on the other one, in such a way that the joint between the layers is at $5 \mathrm{~mm}$ from this support so that the mould edge will be at $10 \mathrm{~mm}$ (Fig. 2).

The joint needs to be far enough from the support to allow the specimen to break readily along the plane of the bond, but the distance must be small so that there is no bending moment in the test. A distance of $5 \mathrm{~mm}$ was decided on based on the experience of Uzan et al. ${ }^{3}$ In their direct shear test a horizontal force was applied to a dual-layer system consisting of prismatic specimens positioned at this distance from the bond between the layers.

The loading piston is located over the metallic mould, $10 \mathrm{~cm}$ apart from each support, and a load at a constant deformation speed of $1.27 \mathrm{~mm} / \mathrm{min}$ is applied; the speed is relatively slow, about the order of half of that used by Uzan et al., ${ }^{3}$ four times less than that of $\mathrm{LROP}^{8}$ and 50 times less than that of Leutner. ${ }^{7}$

The test was performed at a slow speed in order to obtain a better appreciation of the adhesive effect of the different emulsions. These differences would be scarcely noticeable if the test were at a faster speed, especially at lower temperatures. The speed selected by Uzan et al. ${ }^{3}$ was $2.5 \mathrm{~mm} / \mathrm{min}$. However, this value is not normally preset in the more basic presses, which are available in most test laboratories, and it must be remembered that one of the reasons for the development of the LCB test is to provide a standard tool for assessing finished work. Thus the speed of $1.27 \mathrm{~mm} /$ min was selected. This was considered slow enough for the purposes of the test and, as it is the speed used for the California bearing ratio (CBR) test, is a default speed setting for the more basic load presses.

The load-deformation data are collected using a suitable computerised data acquisition system. The equation which provides the value of the shear strength on the bond joining both parts is

\begin{tabular}{|l|l|}
\hline T & $\boldsymbol{T}=(\boldsymbol{P} / 2) / S$ \\
\hline
\end{tabular}

where $\boldsymbol{T}$ is the shear strength, $\boldsymbol{P}$ is the maximum failure load and $S$ is the cross-sectional area of the specimen.

Other parameters can also be obtained from the test, such as the shear modulus (the relationship between maximum tension and displacement) or the specific cracking energy (the area below the load-displacement curve divided by the cracking area). These parameters also characterise the adhesive bond between layers. However, only the peak shear strength has been considered so as to facilitate the use of the test for quality control and to minimise the effect of any deformations that could occur in the mixtures near the supports during the test.

\subsection{Testing of extracted cores: control of tack coats}

The same test procedure can be applied to cores extracted from the road. The cores, which consist of at least two layers, are placed in a cylindrical clamp which is tightened using bolts to hold them in the required position, in a similar way to the laboratory moulded specimens (Fig. 3).
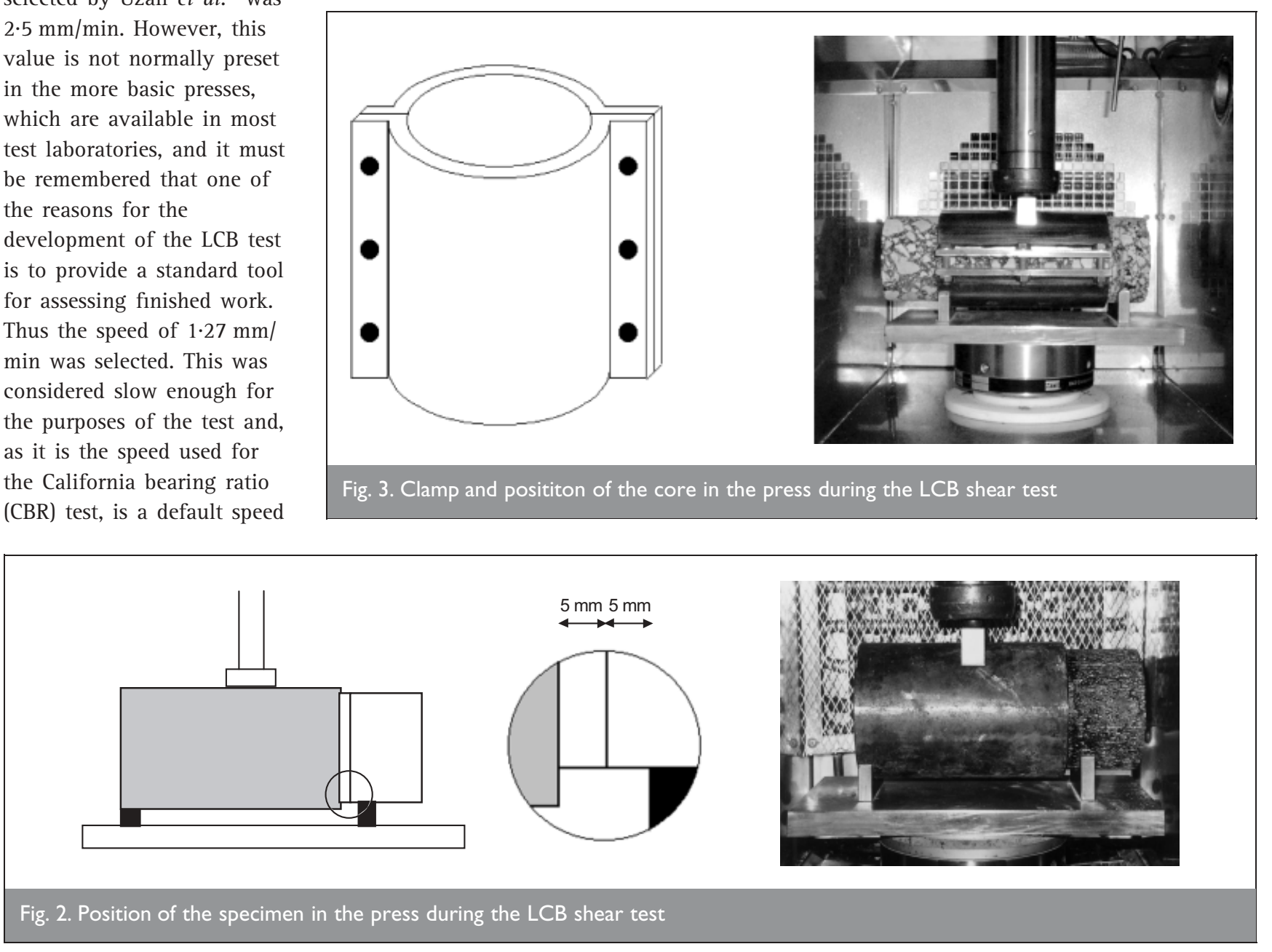
If the test temperature is different from the room temperature, the specimens are placed in a temperature-controlled environment (oven or refrigerator) until they reach the required temperature. Although it is advisable to have a test press with a temperature-controlled chamber in order to keep the test temperature constant, the test is performed at such a speed that there will be no significant temperature change if the test is performed at room temperature.

The test method also has made it possible for cores and laboratory specimens to be tested at the desired temperature after being submerged in water for a certain time at a specified temperature.

Potti et al. ${ }^{14}$ have compared the LCB test with other testing methods to assess the adhesion of adhesive tack coats.

- The elcometer (normally used to measure the adhesion of coatings and paints in construction materials) which, by using the principle of measurement based on the deformation of strain gauges, determines the force of traction necessary to remove the coating to be tested from its application base.

- The direct tensile test (known as endacma) on cylindrical two-layer specimens made up of two layers.

- The LCB method.

They concluded that the cohesive energy, determined by the LCB adhesion test, is the most suitable parameter for assessing adhesion between layers.

\subsection{Test variability}

In earlier stages of the development of the test, the variability of the results for a wide range of test conditions (different types of emulsions, different test temperatures, testing in dry conditions and after immersion) was studied by the authors.

Fig. 4 shows the confidence limits of the test results for different levels of shear strength. These confidence limits have been calculated as: $m \pm 1.96 \sigma$, where $m$ is the mean and $\sigma$ is the standard deviation, calculated from three individual results.

The variation coefficients are low, but it is important to consider that these tests were carried out on specimens manufactured in the laboratory. The mixture for the upper and

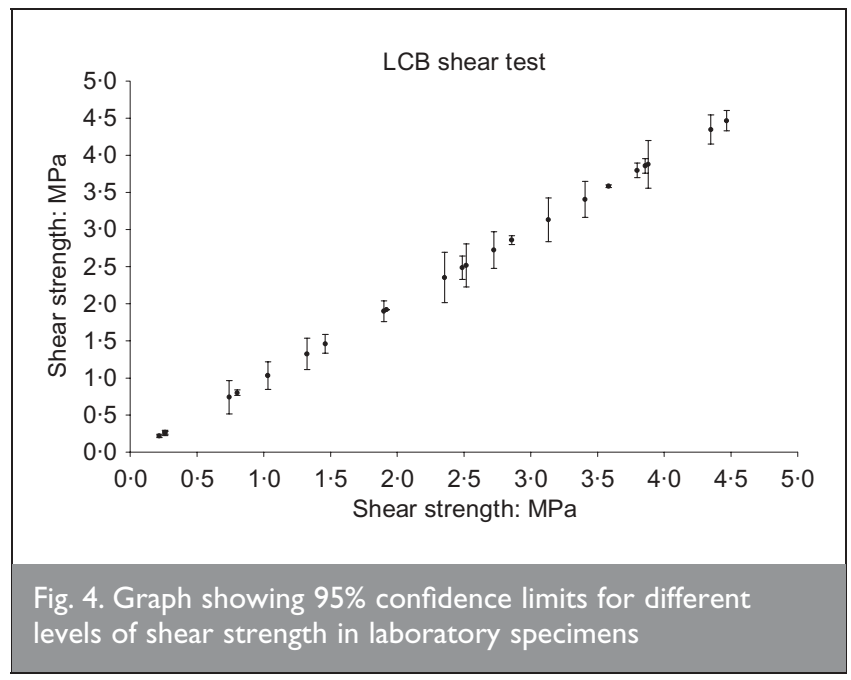

lower layers is always the same, so the emulsion used for the tack coat is the only variable. In this case, the variation coefficients for the shear strength of laboratory specimens range between $1 \%$ and $9 \%$.

On the other hand, the results obtained on cores extracted from different roads have higher dispersion because the mixture of the upper and lower layer is not always the same (different mixture composition, bitumen type, bitumen content, compaction, voids content, etc.). Fig. 5 shows the confidence limits calculated in the same way. In this case, the variation coefficients range between $6 \%$ and 25\%, where the number of individual results to calculate mean values ranges between 2 and 5.

\section{APPLICATION OF THE LCB SHEAR TEST TO ASSESS THE EFFECT OF HEAT-ADHESIVE EMULSIONS}

Two hard residual heat-adhesive emulsions have been used in this study: a conventional one and one modified with polymers, which will be called E1-h and E2-h-m, respectively. The third emulsion is a conventional type ECR-1 (E3), manufactured with a 150/200 penetration bitumen, and this will be used as reference. The characteristics of these three emulsions are shown in Table 1. The quantity of emulsion used for the tack coats was $350 \mathrm{~g} / \mathrm{m}^{2}$ in all three cases.

The quantity of emulsion normally used in Spain for adhesive tack coats is about $400 \mathrm{~g} / \mathrm{m}^{2}$. However, as heat-adhesive emulsions normally have a higher bitumen content than conventional emulsions, this value falls slightly to a fixed $350 \mathrm{~g} / \mathrm{m}^{2}$.

In the first part of the study the bond given by these emulsions was determined by testing specimens manufactured in the laboratory. In the second stage, this same property was determined by testing cores extracted from three experimental sections of a constructed pavement, where the same emulsions and the same dosages were used.

\section{I. Testing specimens}

For the upper and lower layers of the specimens a dense mixture manufactured with calcareous aggregates was used with a maximum size of $8 \mathrm{~mm}$ and a bitumen content of 5.0\% on aggregates. The bitumen was modified with polymers, type

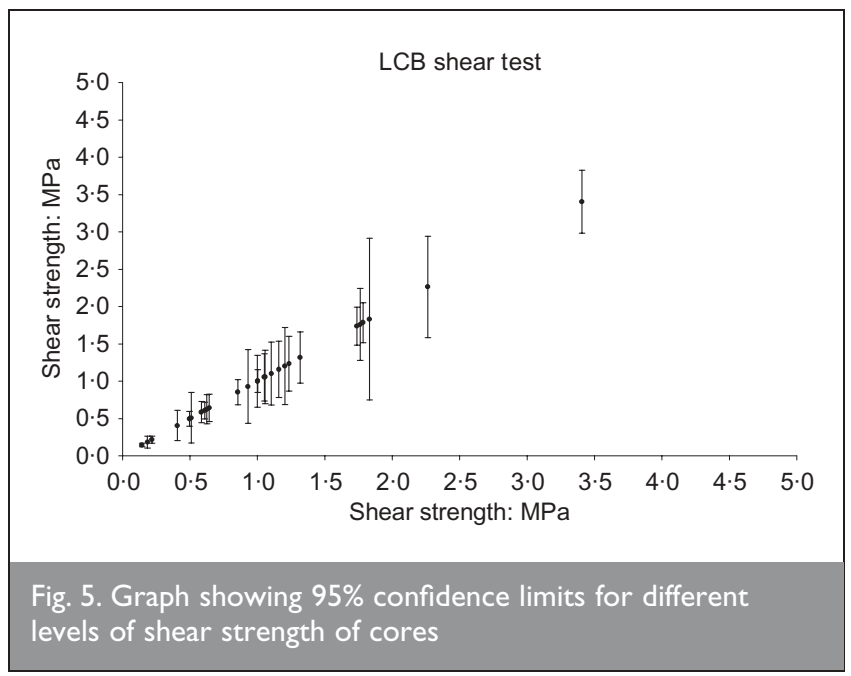




\begin{tabular}{|lccc|}
\hline Properties & EI-h & E2-h-m & E3 \\
\hline Passing 0.5 mm (\%) & 0 & 0 & 0 \\
Viscosity SSF, at $25^{\circ} \mathrm{C}(\mathrm{s})$ & 37 & - & 32 \\
Viscosity SSF, at $50^{\circ} \mathrm{C}(\mathrm{s})$ & - & 31 & - \\
Sedimentation (\%) & $2 \cdot 5$ & 4 & $2 \cdot 5$ \\
Distillation residue (\%) & 60.0 & - & $59 \cdot 0$ \\
Penetration $(0.1 \mathrm{~mm})$ & 24 & - & 165 \\
Fraass brittle point $\left({ }^{\circ} \mathrm{C}\right)$ & +1 & - & -15 \\
Evaporation residue at $163^{\circ} \mathrm{C}(\%)$ & - & $64 \cdot 0$ & - \\
Penetration $(0.1 \mathrm{~mm})$ & - & 30 & - \\
R\&B $\left({ }^{\circ} \mathrm{C}\right)$ & - & $64 \cdot 0$ & - \\
Elastic recovery at $40^{\circ} \mathrm{C}(\%)$ & - & 22 & - \\
Fraass brittle point $\left({ }^{\circ} \mathrm{C}\right)$ & - & -4 & - \\
\hline Table I. Characteristics of the emulsions studied & \\
\hline
\end{tabular}

BM-1, collected according to article 215 of the Spanish technical specifications. ${ }^{15}$

The tests were performed at a constant speed of deformation of $1.27 \mathrm{~mm} / \mathrm{min}$, both in dry conditions and after immersion in water at $30^{\circ} \mathrm{C}$ for $48 \mathrm{~h}$. The test temperature was varied from low temperatures, where the emulsion has a brittle behaviour (especially the hard residual emulsions) to relatively high temperatures, where the residual bitumen is softer, reducing its adhesive properties. The range of test temperatures varied from -15 to $+45^{\circ} \mathrm{C}$.

3.1.1. Dry conditions test. As an example, the average curves obtained for each of the emulsions can be seen in Fig. 6, at a temperature of $+20^{\circ} \mathrm{C}$. The variation of average resistance for each one of them with the temperature is represented in Fig. 7, with the test performed at temperatures of $-15,-5,+5,+20$ and $+45^{\circ} \mathrm{C}$ respectively. Each of the average values has been calculated from three individual results.

When the average values of the peak shear strength shown in Fig. 7 are examined, taking into account the standard error of the test results, some significant differences in the behaviour of the emulsions analysed can be seen.

The first observation is that, for all of the analysed emulsions, the lower the test temperature, the higher the shear strength.

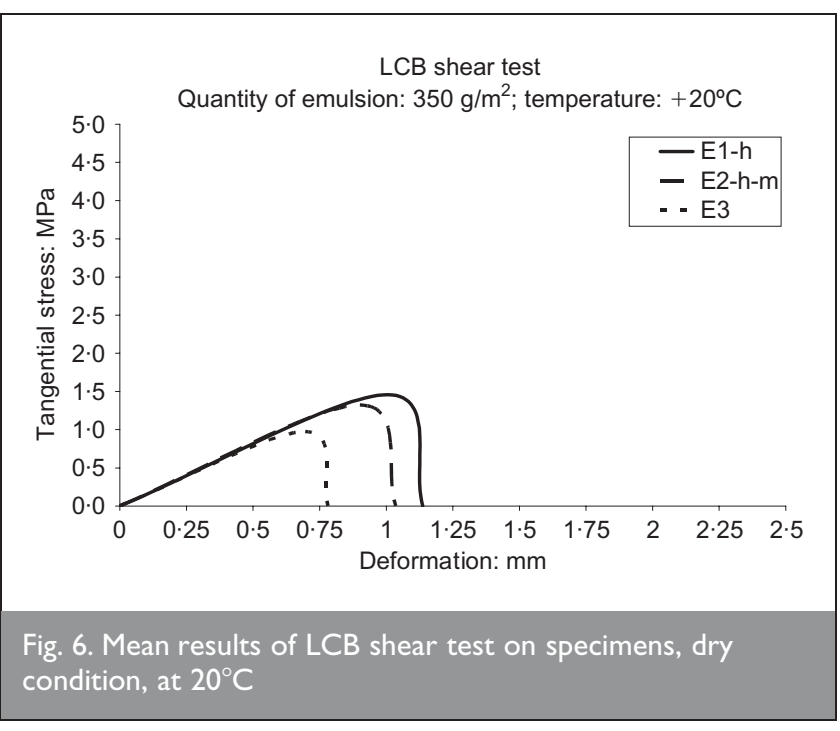

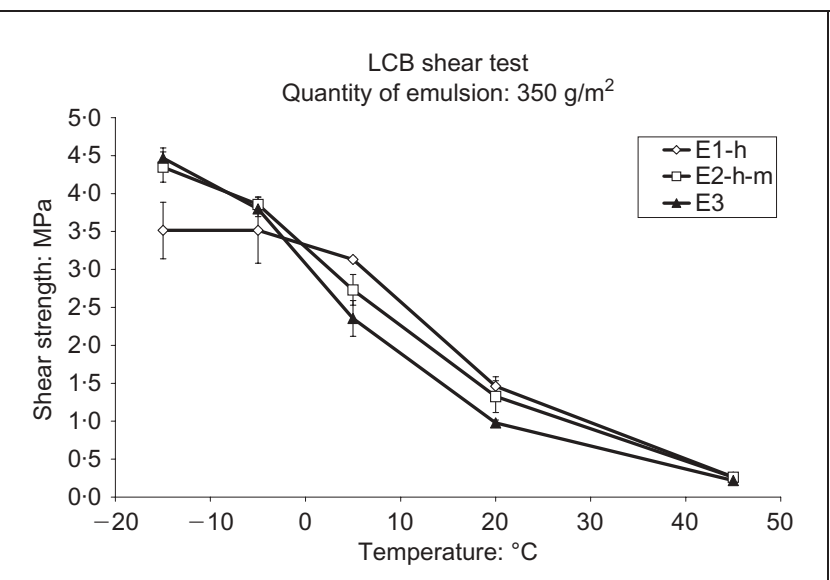

Fig. 7. Mean results of LCB shear test on specimens, dry condition, at different temperatures

However, the increases in the progressive strength that can be seen when lowering the temperature of the tack coat with the E1-h emulsion, stop at $-5^{\circ} \mathrm{C}$, so that the resistance (3.5 MPa) remains the same until $-15^{\circ} \mathrm{C}$. On the other hand, for E3 and E2-h-m emulsions, the resistance continues increasing from $3.8 \mathrm{MPa}$ at $-5^{\circ} \mathrm{C}$ to $4.5 \mathrm{MPa}$ and $4.3 \mathrm{MPa}$, respectively, at $-15^{\circ} \mathrm{C}$. The probable reason for this is that the Fraass brittle point of base bitumens is lower for these last two emulsions: +1 for 13/22 pen bitumen, -4 for the modified bitumen and -15 for the 150/200 pen base bitumens of the emulsions E1-h, E2-h-m and E3, respectively.

From the results obtained at low temperatures it can also be observed that the emulsion with a higher shear resistance is E3, followed very closely by the E2-h-m. The one with the least shear strength is the E1-h emulsion.

At $+5^{\circ} \mathrm{C}$, however, it can be seen that the ranking of the three emulsions on the basis of resistance to shear is reversed. The emulsion that offered more resistance at low temperatures, E3, has less resistance at $+5^{\circ} \mathrm{C}$, while the E1-h now performs better. The shear strength of specimens with E1-h emulsion decreases a little, from $3.50 \mathrm{MPa}$ at $-15^{\circ} \mathrm{C}$ to $3.15 \mathrm{MPa}$ at $+5^{\circ} \mathrm{C}$. On the other hand the E2-h-m and the E3 drop from 3.80 at $-5^{\circ} \mathrm{C}$, to $2 \cdot 75$ and $2 \cdot 35$, respectively, at $+5^{\circ} \mathrm{C}$.

At $+20^{\circ} \mathrm{C}$ the strength values decrease markedly, but the tendency observed at $+5^{\circ} \mathrm{C}$ continues-that is, $1.5 \mathrm{MPa}$ for the E1-h and 1.0 MPa for E3, with an intermediate value for the E2-h-m. Finally, at $+45^{\circ} \mathrm{C}$, the strengths are very low, less than $0 \cdot 25 \mathrm{MPa}$, and no significant differences between the emulsions were observed.

3.1.2. After immersion test. The immersion test was conducted at $+20^{\circ} \mathrm{C}$ after the specimens were immersed in water at $+30^{\circ} \mathrm{C}$ for $48 \mathrm{~h}$. The average resistance obtained under these conditions, with each of the emulsions (Fig. 8), is very similar to that obtained for dry conditions. There is no appreciable decrease in the resistance as a result of the immersion. Besides, the strengths obtained have the same tendency as the ones observed under dry conditions: the conventional emulsion E3 is the least resistant to shear stress, about 1.0 MPa, while the heat-adhesive emulsions E2-h-m and E1-h have slightly higher resistance -1.3 and $1.5 \mathrm{MPa}$, respectively. 


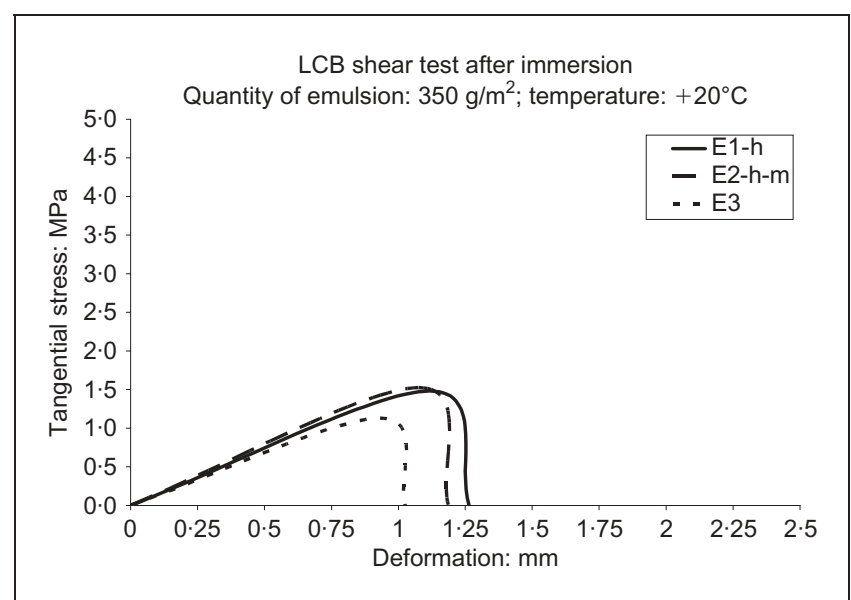

Fig. 8. Mean results of LCB shear test on specimens, after immersion, at $20^{\circ} \mathrm{C}$

\subsection{Testing cores}

These same three emulsions-E1-h, E2-h-m and E3-were applied as tack coats during the construction of the pavement in the C-112 highway, Valladolid, Spain, in three separate sections. They were applied between the wearing and binder courses, made with semi-dense bituminous mixtures of type S-12 and S-20, respectively; both with 60/70 pen bitumen, using the same coat dosage as before $\left(350 \mathrm{~g} / \mathrm{m}^{2}\right)$.

Three months after construction several cores were cut from the pavement and were tested for shear stress resistance at two different temperatures $\left(+20\right.$ and $\left.-15^{\circ} \mathrm{C}\right)$ in dry conditions. The aim was to assess the actual behaviour of the heat-adhesive emulsions at a medium temperature and a low temperature, so that the harder residual bitumen of these emulsions would show fragile behaviour, decreasing the adhesive ability.

As with the laboratory specimens, three cores were tested for each emulsion and for each test temperature.

At $+20^{\circ} \mathrm{C}$, the average resistances obtained from the cores tested (Fig. 9) show the same trend as those obtained with specimens tested at the same temperature. Thus, the emulsion with the greater shear resistance is the conventional heat-

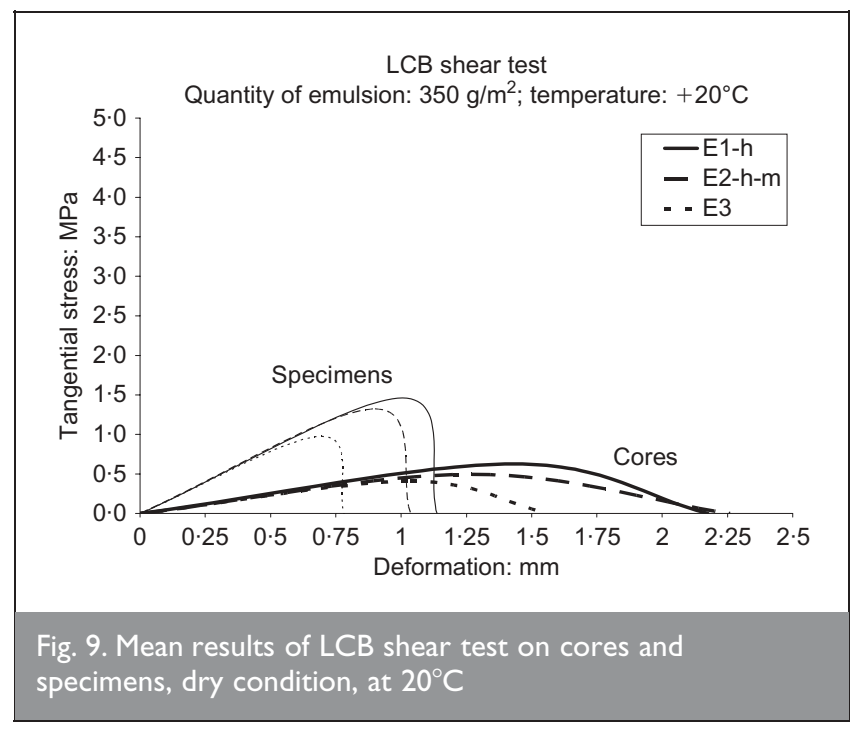

adhesive emulsion (E1-h), followed by the modified heatadhesive emulsion (E2-h-m) and finally, the conventional emulsion (E3).

However, these shear resistances are considerably lower than those obtained from the laboratory specimens. Although it is usual to obtain less resistance from cores than from laboratory specimens, in this case the reduction is more than 50\%. Also, the deformations measured on cores are greater than those obtained on laboratory specimens.

It should also be noted that, as the layers are new, there is a greater amount of bitumen at the interface between the layers of the extracted cores (from the tack coat emulsion and from the mixes that comprise the layers) which means that the cores display a more ductile break, which is particularly noticeable at medium temperatures. Moreover, in some cases a deformation was produced in the mix itself close to the interface due to inadequate compaction during construction. Also, faulty operation of the injectors of the spray bar was detected that prevented a homogeneous spray.

Similar comments can be made when observing the average shear resistances obtained at $-15^{\circ} \mathrm{C}$ (Fig. 10). In this case the resistances obtained for all of the emulsions are very similar, somewhat higher for the modified heat-adhesive emulsion, but substantially lower than those obtained for the laboratory specimens. On the other hand, as the shear strength is low and the breaking at low temperatures is still fragile, the deformations are lower than those obtained from the specimens tested, unlike those obtained at $+20^{\circ} \mathrm{C}$.

\section{CONCLUSIONS}

In view of the results obtained with the LCB shear test for each one of the emulsions analysed in this study, it is possible to conclude that the heat-adhesive modified emulsion, E2-h-m, performs better, although the differences observed with the other emulsions are not very great.

The conventional heat-adhesive emulsion, E1-h, performs better at medium temperatures, but at low temperatures its greater fragility means it does not achieve the shear resistances of the other emulsions.

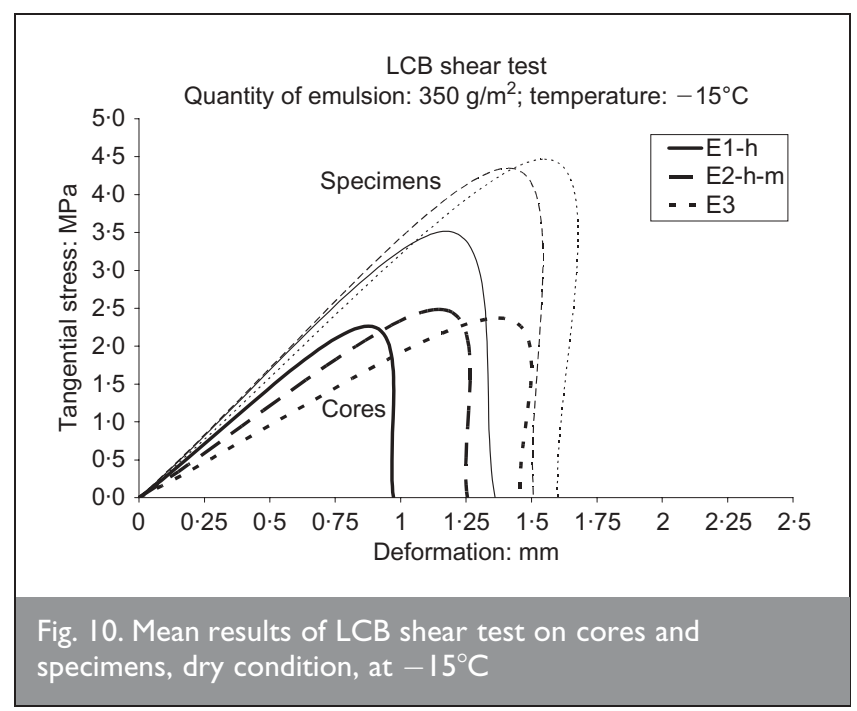


On the other hand, the conventional emulsion E3, manufactured with a softer bitumen than the heat-adhesive ones, displays a high shear resistance at low temperatures, similar to the modified heat-adhesive emulsion, while at medium or high temperatures it has less resistance.

The strengths demonstrated after immersion, at $20^{\circ} \mathrm{C}$, are very similar to those obtained in dry conditions. This means that the degree of adhesion provided by all of the emulsions evaluated is not altered by immersion in water.

Moreover, the results of tests on cores confirm the trend observed with the laboratory specimens, although the shear resistance of the cores was much lower than that of the laboratory specimens due to the effect of bitumen from the mixes and to the faulty performance of the spray bar.

\section{ACKNOWLEDGEMENT}

The authors would like to acknowledge the financial assistance for this study from the Ministerio de Ciencia y Tecnología, Spain (MAT2000-0230-P4-03).

\section{REFERENCES}

1. Tschegg E. K., Kroyer G., TAN D., Stanzl-Tschegg S. and LITZKA J. Investigation of bonding between asphalt layers on road construction. Journal of Transportation Engineering, 1995, 121, No. 4, 309-316.

2. Tschegg E. K. An efficient fracture test method for bituminous materials and layer bonds. In Mechanical Tests for Bituminous Materials (H. Di BENEDETTO and L. FRANCKEN (eds)). RILEM, Rotterdam, 1997, pp. 405-411.

3. UZAN J., LIVNEH M. and ESHED Y. Investigation of adhesion properties between asphaltic-concrete layers. Asphalt Paving Technology, 1978, 47, 495-521.

4. Caltabiano M. A. and Brunton J. M. Reflection cracking in asphalt overlays. Asphalt Paving Technology, 1991, 60, 310-332.

5. Hughes D. A. Polymer Grid Reinforcement of Asphalt Pavements. PhD Thesis. Nottingham University, 1986.

6. MolenAar A. A. A. Evaluation of pavement structure with emphasis on reflective cracking. In Reflective Cracking in Pavement. State of the Art and Design Recommendations (J. M. Rigo, R. DEgeimbre and L. FrancKen (eds)). RILEM, Cambridge, 1993, pp. 21-48.

7. ForschungSgESELLSCHAFT FÜr STRAßEN- UND VERKEHRSWESEN. Prüfung des Schichtenverbundes nach Leutner. Probenahme und Durchführung der Prüfung, 1998.

8. Abdo J., Baroin L., Batut X., Grand E., Guiraud P., Huvelin B., Potier J. M., Sainton A., Sigwald B., Soufflet J. P., TABAILlon C. and Monneraye P. L'étude du collage d'un BBTM sur un revêtement en betón âgé de quelques jours. Un nouveau project de recherche fruit du partenariat CIMBETON/SPECBEA/SNBPE. Revue Générale des Routes, 2001, No. 798, 22-28 (in French).

9. BRITISH BOARD OF AGRÉMENT. Guideline Document for the Assessment and Certification of Thin Surfacing Systems for Highways. British Board of Agrément, Watford, 1998, Working Draft 2, 19-22.

10. KHWEIR K. and FORDYCE D. Influence of layer bonding on the prediction of pavement life. Proceedings of the Institution of Civil Engineers-Transport, 2003, 156, 73-83.

11. BORRÁs J. M. Efecto del uso de ligantes modificados en los riegos de adherencia. Tesina de especialidad. Degree thesis, ETSICCPB, Universidad Politécnica de Cataluña, Barcelona, 1999 (in Spanish).

12. Miró R., PÉREZ F., SOTO J.A. and FERNÁNDEZ M. Evaluación de la adherencia entre capas mediante la aplicación del nuevo ensayo de corte LCB. Report de recerca, Universidad Politécnica de Cataluña, Barcelona, 2002 (in Spanish).

13. CEDEX. NLT-161/84 Resistencia A compresión simple de mezclas bituminosas. Normas NLT-Ensayos de Carreteras. Cedex, Madrid, 1984 (in Spanish).

14. Potti J. J., PeÑA J. L. and GUZMÄN F. Emulsiones termoadherentes para riegos de adherencia. Carreteras, 2003, 128, 17-26 (in Spanish).

15. Ministerio De Fomento. Pliego de Prescripciones Técnicas Generales para Obras de Carreteras y Puentes. Orden de 27 de Diciembre de 1999, Conglomerantes hidráulicos y ligantes hidrocarbonados. Ministerio de Fomento, Madrid, 1999 (in Spanish).

Please email, fax or post your discussion contributions to the secretary by I August 2005: email: journals@ice.org.uk; fax: +44 (0)20 665 2294; or post to Journals Department, Institution of Civil Engineers, I-7 Great George Street, London SWIP 3AA. 